\title{
Theory of an all-carbon molecular switch
}

\author{
R. Gutierrez, ${ }^{1}{ }^{*}$ G. Fagas,${ }^{2}$ G. Cuniberti, ${ }^{2}$ F. Grossmann, ${ }^{1}$ R. Schmidt, ${ }^{1}$ and K. Richter ${ }^{3}$ \\ ${ }^{1}$ Institute for Theoretical Physics, Technical University of Dresden, D-01062, Germany \\ ${ }^{2}$ Max Planck Institute for the Physics of Complex Systems, D-01187 Dresden, Germany \\ ${ }^{3}$ Institute for Theoretical Physics, University of Regensburg, D-93040 Regensburg, Germany
}

(Received 8 August 2001; published 28 February 2002)

\begin{abstract}
We study electron transport across a carbon molecular junction consisting of a $\mathrm{C}_{60}$ molecule sandwiched between two semi-infinite metallic carbon nanotubes. It is shown that the Landauer conductance of this carbon hybrid system can be tuned within orders of magnitude not only by varying the tube- $\mathrm{C}_{60}$ distance, but more importantly at fixed distances by (i) changing the orientation of the Buckminsterfullerene or (ii) rotating one of the tubes around its cylinder axis. Furthermore, it is explicitly shown that structural relaxation determines qualitatively the transmission spectrum of such devices.
\end{abstract}

DOI: 10.1103/PhysRevB.65.113410

Driven by advances in chemical synthesis, scanning probe microscopy and break junction techniques, the seminal idea of using molecular scale conductors as active components of electronic devices ${ }^{1}$ has received a new impetus in recent years. $^{2-4}$ Rectification and negative differential resistance could already be demonstrated on the nanoscale. ${ }^{3}$ However, mechanical, electrical or electromechanical switching devices and transistors still include components of mesoscopic dimensions. ${ }^{3}$

One of the most intensively studied molecules in the field is the fullerene $\mathrm{C}_{60}$, whose electronic transport properties were measured and manipulated by scanning tunneling microscopy (STM) techniques. ${ }^{5}$ Several examples of fullerenebased devices have been investigated both experimentally ${ }^{6-9}$ and theoretically. ${ }^{10-13}$ Suggested mechanisms to control the conductance at a single $\mathrm{C}_{60}$ molecular junction are either of electromechanical nature (utilizing an STM tip to compress the molecule ${ }^{5,10}$ ) or based on charge transfer (controlled by a gate potential in a three-terminal geometry ${ }^{12}$ ). The first mechanism has already been realized experimentally, although the theoretical explanation of the observed effect is still inconclusive. ${ }^{10,11}$ The second approach runs into the difficulty of applying a gate terminal at very short length scales. Experimentally there is some evidence of a third mechanism that changes the conductance properties of $\mathrm{C}_{60}$ by altering the orientation of the molecule with respect to a substrate. This mechanism could explain the observed changes in contrast of STM pictures of molecular $\mathrm{C}_{60}$ layers on gold surfaces. . $^{14,15}$

Other prime targets as possible building blocks for nanoscale electronics devices are single- and multiple-wall carbon nanotubes $(\mathrm{CNTs}) .{ }^{16}$ This owes to their exceptional electronic and structural properties, that have been extensively studied over the decade following their discovery. It has been demonstrated that tubes can act as both a wiring system ${ }^{17}$ and active device elements. ${ }^{17-21}$ In the latter a gate voltage was applied to manipulate the conductance. Recently, it has also been shown that if CNTs are placed on a graphite substrate, the interface resistance can be tuned by changing the tube orientation on the graphite plane in- or out-of-registry, with an atomic force microscope tip. ${ }^{22}$ Similar effects have been studied theoretically for a junction of two CNTs in a $\pi$-electron tight-binding formulation. ${ }^{23}$
PACS number(s): 73.63.Fg, 61.46.+w, 71.15.Ap, 72.80.Rj

In this paper, we propose an alternative switching device entirely on the nanoscale which combines the unique features of both $\mathrm{C}_{60}$ and CNTs, in a CNT- $\mathrm{C}_{60}$-CNT hybrid system (see Fig. 1). The electronic transport in this pure-carbon electronics setup is studied in the Landauer formalism ${ }^{24}$ using Green function techniques combined with a density functional theory (DFT) based approach. We show that an unexpectedly large variation of the conductance over three orders of magnitude can be achieved by either changing the orientation of $\mathrm{C}_{60}$, or by rotating the nanotubes around the symmetry axis at a fixed tube- $\mathrm{C}_{60}$ distance. In addition, it is demonstrated that the conductance of such molecular devices strongly depends on the structural relaxation of the junction.

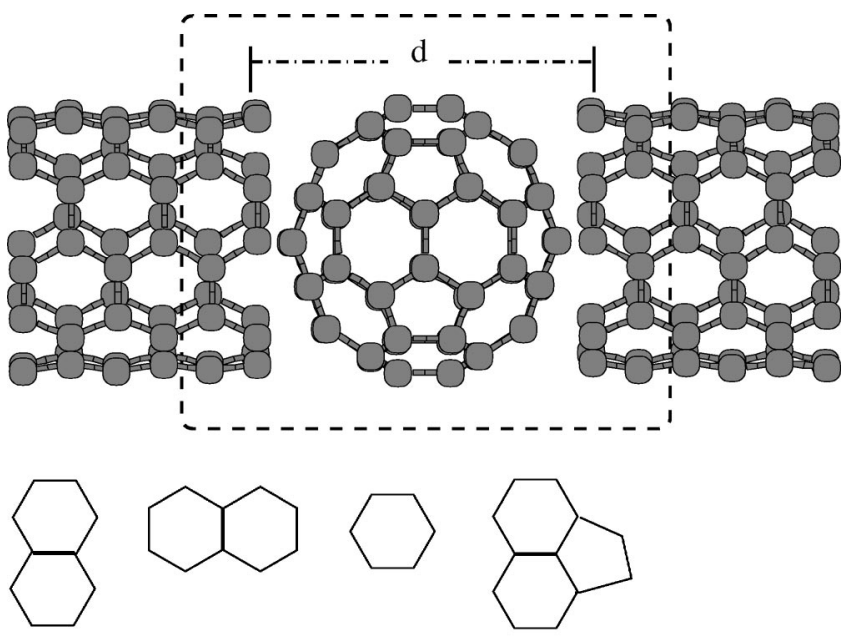

(1)

(2)

(3)

(4)

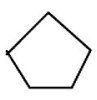

(5)

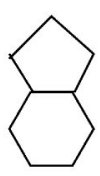

(6)

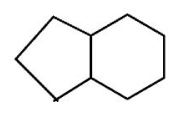

(7)
FIG. 1. Geometric configuration of the molecular junction discussed in this paper. $\mathrm{A}_{60}$ molecule bridges two $(5,5) \mathrm{CNTs}$. The dashed-line frame encloses the scattering region. The lower panel represents schematically the different orientations of $\mathrm{C}_{60}$ with respect to the surface cross sections of the nanotubes [e.g., the upper panel geometry corresponds to orientation (1)]. 
In the Landauer approach, the conductance of the system is related to an independent-electron elastic scattering problem, with the transmission function $T(E)$ at the Fermi energy $E_{\mathrm{F}}$ determining the two-terminal, linear-response conductance in the zero temperature limit

$$
g=\frac{2 e^{2}}{h} T\left(E_{\mathrm{F}}\right) .
$$

The Green function method is employed to calculate $T(E)$ from

$$
T(E)=\operatorname{Tr}\left[\Gamma_{\mathbf{L}} \mathbf{G}^{\mathbf{r}} \Gamma_{\mathbf{R}} \mathbf{G}^{\mathbf{a}}\right] .
$$

In a nonorthogonal basis representation, the retarded and advanced Green functions $\mathbf{G}^{\mathbf{r}, \mathbf{a}}$ are determined by solving the finite dimensional matrix equation

$$
\left(E \mathbf{S}-\mathbf{H}-\Sigma_{\mathbf{L}}-\Sigma_{\mathbf{R}}\right) \mathbf{G}=\mathbf{1},
$$

which can be derived using a partitioning technique for nonorthogonal bases. ${ }^{25}$ Here, $\mathbf{H}$ and $\mathbf{S}$ are the molecular Hamiltonian and the overlap matrix, respectively. The self energies $\Sigma_{\mathbf{L}}\left(\Sigma_{\mathbf{R}}\right)$ depend on the coupling matrix elements between the scattering region and the left (right) lead and on the lead Green functions. Their imaginary parts determine the matrices $\Gamma_{\mathbf{L}, \mathbf{R}}=i\left[\Sigma_{\mathbf{L}, \mathbf{R}}-\Sigma_{\mathbf{L}, \mathbf{R}}^{\dagger}\right]$. $^{24}$

In the following, we characterize the electronic structure of the molecule and the leads as well as the lead-molecule coupling within an approximate DFT-parametrized framework. The method relies on a nonorthogonal linear combination of (valence) atomic orbitals (LCAO) ansatz and it has been previously applied to study dynamical, ${ }^{28}$ structural, ${ }^{29}$ and electronic transport ${ }^{26,27}$ properties of a large class of materials. The Green functions of the tubes have been calculated by using a recursive technique. ${ }^{30}$ Structural optimization is performed by using conjugate-gradient techniques and taking into account a cluster consisting of the $\mathrm{C}_{60}$ cage and six unit cells of the CNT on either side of it. To simulate the effect of semi-infinite leads we allowed only the fullerene and the first unit cells of the tubes (nearest to $\mathrm{C}_{60}$ ) to relax, thus defining the scattering region of Fig. 1.

We study a single $\mathrm{C}_{60}$ molecule bridging two single-wall metallic $(5,5)$ nanotubes, which are taken symmetric with respect to the plane through the center of mass of $\mathrm{C}_{60}$ and perpendicular to the CNT cylinder axes (see upper panel of Fig. 1 for a typical unrelaxed structure). The tubes act as donor and acceptor electron reservoirs. First, we investigate the distance dependence of the conductance for a given configuration. The central aim of this letter, however, is to exploit the sensitivity of electron transport to the topology of the molecule/electrode interface ${ }^{31}$ in the proposed system. To this end several possible orientations of the $\mathrm{C}_{60}$ [depicted by the polygon(s) facing the tube symmetry axis in the lower panel of Fig. 1] have been considered. Finally, the dependence of the conductance on the rotation of one of the tubes around the symmetry axis at a fixed orientation of $\mathrm{C}_{60}$ is investigated.

The energy dependence of the total transmission at three different tube-tube separations $d$ is shown in Fig. 2 for the case of the (relaxed) configuration (5). ${ }^{32}$ Large variations in

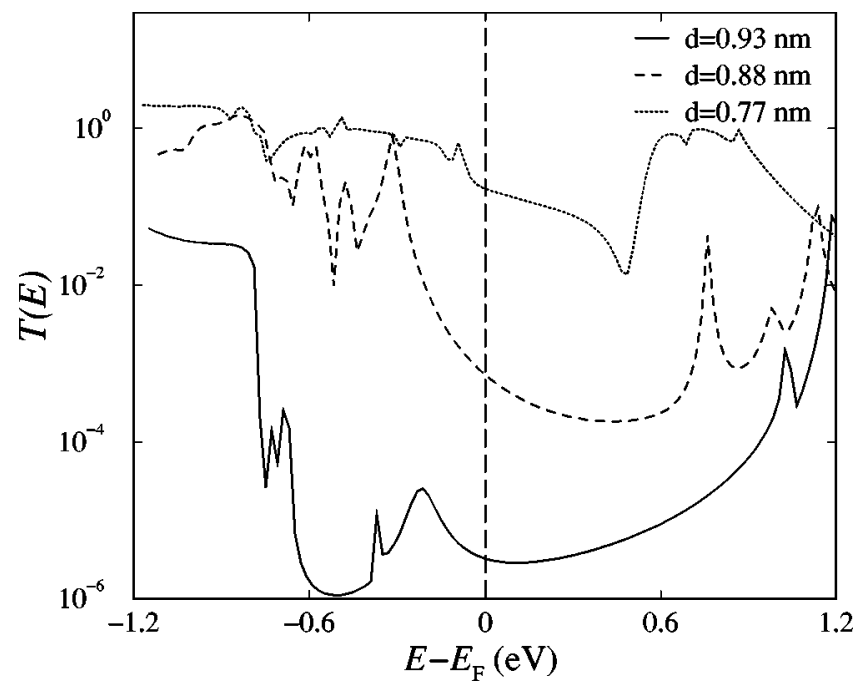

FIG. 2. Transmission spectra for the relaxed configuration with orientation (5) (see Fig. 1) for different distances $d$ between the nanotubes.

the transmission of up to 5 orders of magnitude are found, similar to those reported in Ref. 11 for the case of a pointlike STM-tip approaching a $\mathrm{C}_{60}$ molecule. A reduction of $d$ increases the molecule-lead coupling and, therefore, leads to a strong broadening and shifting of the resonances, as can be seen in Fig. 2. For very short separations the cage geometry of $\mathrm{C}_{60}$ distorts and no resonances are resolved any more. ${ }^{33}$ The molecule becomes highly transparent $[T(E) \sim 1]$ over a wide energy range around the Fermi level. We stress that the presented results for the distance dependence are very sensitive to the inclusion of structural relaxation and strongly differ from results for unrelaxed structures (not shown).

A key question is what happens for a fixed distance between the molecule and the tubes but for different orientations of $\mathrm{C}_{60}$ (see lower panel of Fig. 1) with the inclusion of relaxation. Such dependence is displayed in Fig. 3 for both relaxed and unrelaxed molecular junctions. Surprisingly, at fixed distance, just an atomic scale rotation of the highly symmetric $\mathrm{C}_{60}$ molecule induces a large variation of the transmission at the Fermi energy by several orders of magnitude. This is shown in Fig. 3(b) for three of the seven different orientations with maximum, minimum, and one intermediate value of $T\left(E_{\mathrm{F}}\right)$. As can be seen in Fig. 3(a), neglecting relaxation decisively influences the transmission properties of the molecular junction. This shows up as a much different and less smooth behavior of the transmission. The qualitative difference is related to the rehybridization and/or partial saturation of the dangling bonds on the CNT surface upon relaxation. It is interesting to note that such a dramatic influence of the relaxation on the conductance has not been reported before for this class of systems. For instance, the relaxation of a CNT-CNT junction in-registry changes the resistance only quantitatively rather than qualitatively. ${ }^{23}$

The results for the relaxed structures reveal that, at the Fermi energy, the pentagon configuration (5) has a transmission lower by about three orders of magnitude than configuration (4). This fact could be exploited in an electronic 


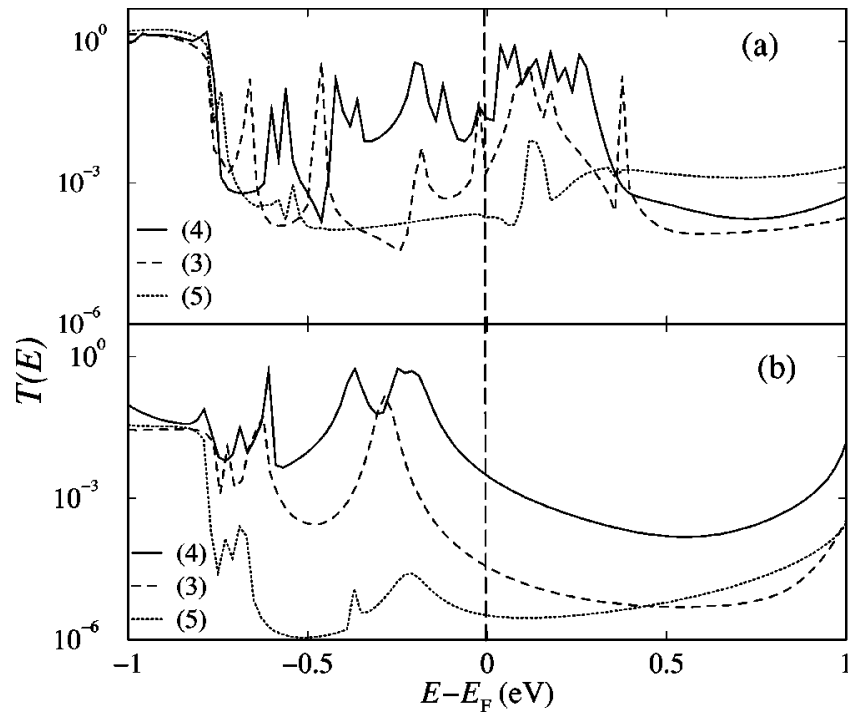

FIG. 3. Transmission results for both unrelaxed (upper panel) and relaxed (lower panel) configurations. $d$ is fixed at $0.93 \mathrm{~nm}$. Numbers indicate different molecular orientations as depicted in the lower panel of Fig. 1.

switching device on the nanoscale, as manipulation of fullerenes by using STM or atomic force microscope tips is becoming a standard technique in the field. ${ }^{8,22}$ Furthermore, this effect may also serve to explain the observation of contrast variations in STM images. ${ }^{14,15}$

Different from other calculations which assume aluminum electrodes ${ }^{12,13}$ the absolute values of the transmission found here are small. In the former case, charge transfer from the electrodes onto the LUMO pins the Fermi energy near this resonance. In our purely carbon-based molecular device, however, charge transfer effects are much weaker, as a Mulliken population analysis shows $(\sim 0.1 e)$. Thus, rather than a ballisticlike transport supported by molecular states, tunnelling through the highest occupied molecular orbital (HOMO)-lowest unoccupied molecular orbital (LUMO) gap is the main mechanism of electronic conductance. ${ }^{34}$ The latter is determined by the superposition of the resonance tails and for molecules with highly degenerate HOMO and LUMO manifolds such as the isolated $\mathrm{C}_{60}$, an increase of this overlap can be achieved by lifting the degeneracy. ${ }^{6,10}$ In our calculations, coupling to the leads changes the electronic structure of the fullerene in a similar fashion. However, our results suggest additional competing effects. The wave function overlap between components at a molecular junction which includes nanoelectrodes strongly depends on the exact atomic configuration ${ }^{31}$ and, hence, the shape and the position of molecular resonances. Moreover, unsaturated dangling bonds introduce additional resonant states within the HOMO-LUMO gap of the isolated $\mathrm{C}_{60} \cdot{ }^{35}$ Note that such states are asymmetrically coupled to the leads. In general, this leads to a value of the conductance at resonance smaller than the quantum unit $\left(2 e^{2} / h\right)$.

In addition to the rotation of $\mathrm{C}_{60}$ in between the tubes, we have also studied the dependence of the conductance upon

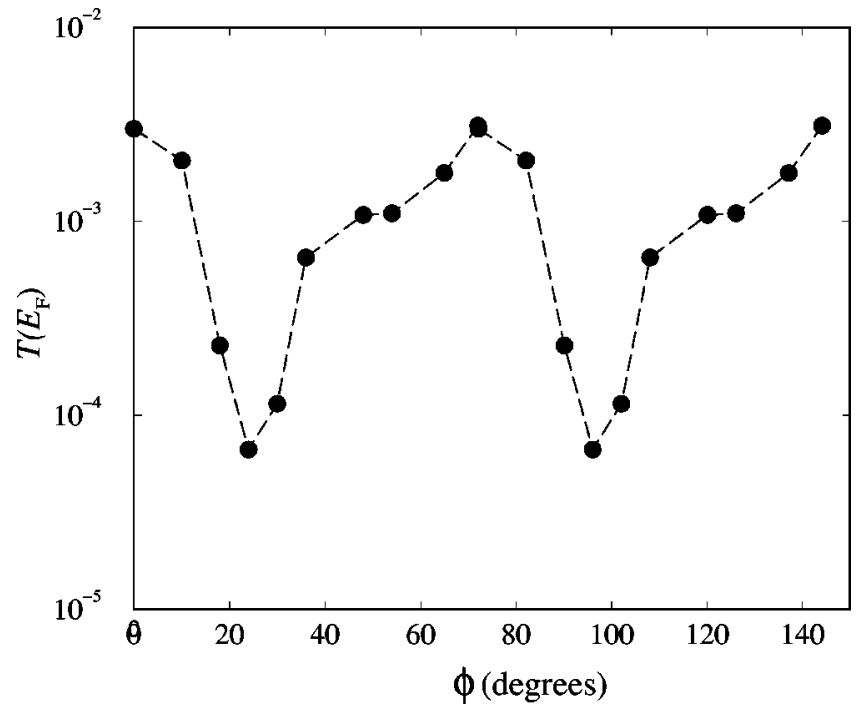

FIG. 4. Transmission at the Fermi energy as a function of the rotation angle $\phi$ of one of the nanotubes around the direction of current transport. The nanotube separation $d$ is $0.93 \mathrm{~nm}$ and $\mathrm{C}_{60}$ has orientation (4) of Fig. 1.

rotation of one of the carbon nanotube electrodes around the axis of current transport. This destroys the high symmetry of the CNT subsystem. As shown in Fig. 4, periodic features in the conductance are found and, again, variations of several orders of magnitude can be observed at the Fermi energy. The rotation angle $\phi=0^{\circ}$ case corresponds to the Fermi level value of the transmission function for orientation (4) in Fig. 3. From this initial, relatively high conducting situation, the transmission can be tuned down by two orders of magnitude by rotating one CNT with respect to the other by $24^{\circ}$. The period of the oscillation of the conductivity is $72^{\circ}$, reflecting the symmetry of the tubes.

In conclusion, we have studied a fullerene-based nanobridge of a $\mathrm{C}_{60}$ molecule sandwiched between two semiinfinite carbon nanotubes in the Landauer formalism. We have found that in this pure carbon molecular junction the conductance is dominated by tunnelling through the HOMOLUMO gap. Most importantly, the transmission can be efficiently controlled by rotations of the $\mathrm{C}_{60}$ molecule and/or one of the nanotubes, thus making this carbon hybrid system a possible candidate for a nano-electronic switching device. Furthermore, we have shown that structural relaxation turns out to have a decisive influence on the electronic transport properties and may not be neglected in the modelling of such nanoscale molecular junctions.

This research was supported by the "Deutsche Forschungsgemeinschaft" through the Forschergruppe "Nanostrukturierte Funktionselemente in makroskopischen Systemen." R.G. gratefully acknowledges financial support by the "Sächsische Ministerium für Wissenschaft und Kunst." G.C. research at MPI is sponsored by the Schlössmann Foundation. F.G. has benefitted from valuable discussions with T. Fritz. 
*Electronic address: gutie@theory.phy.tu-dresden.de

${ }^{1}$ A. Aviram and M.A. Ratner, Chem. Phys. Lett. 29, 277 (1974).

${ }^{2}$ J. Chen, M.A. Reed, A.M. Rawlett, and J.M. Tour, Science 286, 1550 (1999).

${ }^{3}$ C. Joachim, J.K. Gimzewski, and A. Aviram, Nature (London) 408, 541 (2000).

${ }^{4}$ A. Nitzan, Annu. Rev. Phys. Chem. 52, 681 (2001).

${ }^{5}$ C. Joachim, J.K. Gimzewski, R.R. Schlittler, and C. Chavy, Phys. Rev. Lett. 74, 2102 (1995).

${ }^{6}$ C. Joachim and J.K. Gimzewski, Chem. Phys. Lett. 265, 353 (1997).

${ }^{7}$ D. Porath, Y. Levi, M. Tarabiah, and O. Millo, Phys. Rev. B 56, 9829 (1997).

${ }^{8}$ C. Zeng, H. Wang, B. Wang, and J.G. Hou, Appl. Phys. Lett. 77, 3595 (2000).

${ }^{9}$ H. Park et al., Nature (London) 407, 57 (2000).

${ }^{10}$ C. Joachim, J.K. Gimzewski, and H. Tang, Phys. Rev. B 58, 16 407 (1998).

${ }^{11}$ M. Paulsson and S. Stafström, J. Phys.: Condens. Matter 11, 3555 (1999).

${ }^{12}$ J. Taylor, H. Guo, and J. Wang, Phys. Rev. B 63, 121104(R) (2001).

${ }^{13}$ J.J. Palacios, A.J. Peréz-Jiménez, E. Louis, and J.A. Vergés, Nanotechnology 12, 160 (2001).

${ }^{14}$ L. Cepek et al., Surf. Sci. 454, 766 (2000).

${ }^{15}$ M. Stöhr et al., J. Funct. Mater. 11, 175 (2001).

${ }^{16}$ P.L. McEuen, Phys. World 13, 31 (2000).

${ }^{17}$ T. Rueckes et al., Science 289, 94 (2000).

${ }^{18}$ R. Martel et al., Appl. Phys. Lett. 73, 2447 (1998).

${ }^{19}$ H.W.C. Postma et al., Science 293, 76 (2001).

${ }^{20}$ H. Watanabe, C. Manabe, T. Shigematsu, and M. Shimizu, Appl. Phys. Lett. 78, 2928 (2001).
${ }^{21}$ C. Thelander, M.H. Magnusson, K. Deppert, L. Samuelson, P.R. Poulsen, J. Nygard, and J. Borggreen, Appl. Phys. Lett. 79, 2106 (2001).

${ }^{22}$ S. Paulson et al., Science 290, 1742 (2000).

${ }^{23}$ A. Buldum and J.P. Lu, Phys. Rev. B 63, 016140(R) (2001).

${ }^{24}$ S. Datta, Electronic Transport in Mesoscopic Systems (Cambridge University Press, Cambridge, 1995).

${ }^{25}$ S. Priyadarshi, S. Skourtis, S.M. Risser, and D.N. Beratan, J. Chem. Phys. 104, 9473 (1996).

${ }^{26}$ R. Gutierrez, F. Grossmann, O. Knospe, and R. Schmidt, Phys. Rev. A 64, 013202 (2001).

${ }^{27}$ G. Cuniberti, R. Gutierrez, G. Fagas, F. Grossmann, K. Richter, and R. Schmidt, Physica E (to be published).

${ }^{28}$ O. Knospe, G. Seifert, and R. Schmidt, Adv. Class. Traj. Meth. 4, 153 (1999).

${ }^{29}$ D. Porezag, Th. Frauenheim, and Th. Köhler, Phys. Rev. B 51, 12 947 (1995).

${ }^{30}$ M.P. López-Sancho, J.M. López-Sancho, and J. Rubio, J. Phys. F: Met. Phys. 15, 851 (1985).

${ }^{31}$ G. Fagas, G. Cuniberti, and K. Richter, Phys. Rev. B 63, 045416 (2001).

${ }^{32}$ To determine the Fermi level for each transport geometry, we have chosen a large cluster consisting of the $\mathrm{C}_{60}$ molecule and several carbon nanotube unit cells on either side. Then, for increasing size of the composite, the Hamiltonian matrix was diagonalized and the electronic states were populated appropriately. The resulting converged HOMO was taken as a first approximation to $E_{\mathrm{F}}$.

${ }^{33}$ Apart from this case, in all the investigated configurations the geometries of the $\mathrm{C}_{60}$ cage and the surface of the CNT's do not appreciably deviate from the ideal geometry.

${ }^{34}$ M. Magoga and C. Joachim, Phys. Rev. B 56, 4722 (1997).

${ }^{35}$ R. Gutierrez et al. (unpublished). 